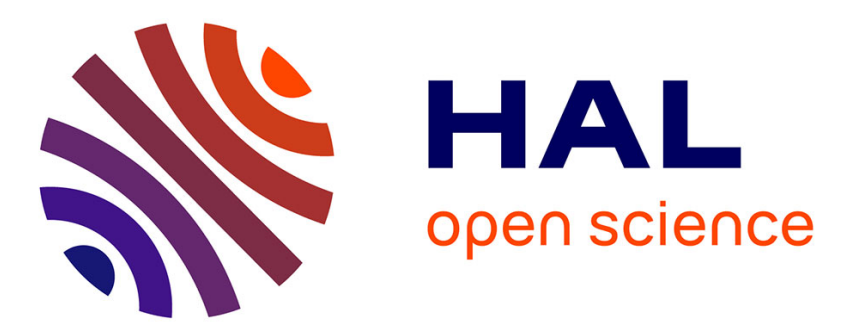

\title{
Using Ground Transportation for Aviation System Disruption Alleviation
}

Lynnette Dray, Isabelle Laplace, Aude Marzuoli, Eric Féron, Antony Evans

\section{To cite this version:}

Lynnette Dray, Isabelle Laplace, Aude Marzuoli, Eric Féron, Antony Evans. Using Ground Transportation for Aviation System Disruption Alleviation. Journal of Air Transportation, 2017, 25 (3), pp. 95-107. 10.2514/1.D0070 . hal-01693255

\section{HAL Id: hal-01693255 \\ https://hal.science/hal-01693255}

Submitted on 2 Feb 2018

HAL is a multi-disciplinary open access archive for the deposit and dissemination of scientific research documents, whether they are published or not. The documents may come from teaching and research institutions in France or abroad, or from public or private research centers.
L'archive ouverte pluridisciplinaire HAL, est destinée au dépôt et à la diffusion de documents scientifiques de niveau recherche, publiés ou non, émanant des établissements d'enseignement et de recherche français ou étrangers, des laboratoires publics ou privés. 


\title{
Using ground transportation for aviation system disruption alleviation
}

\author{
Lynnette Dray*, \\ Energy Institute, University College London, UK WClH ONN \\ Isabelle Laplace ${ }^{\dagger}$ \\ ENAC/Université de Toulouse, Toulouse, France 31055 \\ Aude Marzuoli ${ }^{\ddagger}$ Eric Feron ${ }^{\S}$
}

Georgia Institute of Technology, GA 30332

and

Antony Evans**

Crown Consulting Inc., CA 94035

\begin{abstract}
We investigate whether passenger delays and airline costs due to disruptive events affecting European airports could be reduced by a co-ordinated strategy of using alternative flights and ground transportation to help stranded passengers reach their final destination, using Airport Collaborative Decision Making concepts. Optimising for airline cost for hypothetical disruptive events suggests that, for airport closures of up to 10 hours, airlines could benefit from up to a $20 \%$ reduction in passenger delay-related costs. Mean passenger delay could be reduced by up to $70 \%$, mainly via a reduction in very long delays.
\end{abstract}

Keywords: Aviation system disruption recovery; collaborative decision making; real-time intermodality

\footnotetext{
* Senior Research Associate. 14, Upper Woburn Place, London, WC1H 0NN. Corresponding author, 1.dray@ucl.ac.uk.

${ }^{\dagger}$ Head of the Air Transport Sustainable Growth Program. 7, Avenue Edouard Belin, F-31055, France Cedex 04.

\$ Graduate student. North Avenue, Atlanta, GA 30332.

$\S$ Professor of Aerospace Software Engineering. North Avenue, Atlanta, GA 30332.

**Senior Scientist I. Ames Research Center, M/S 210-6, Moffett Field, CA 94035.
} 


\section{INTRODUCTION}

In its vision for aviation in 2050, the European Commission [1] sets the goal: "90\% of travellers within Europe are able to complete their journey, door-to-door, within 4 hours. Passengers and freight are able to transfer seamlessly between transport modes to reach the final destination smoothly, predictably and on-time."

Historically, metrics for delay and disruption at airports have concentrated on the impact on individual flights rather than passengers. However, as reflected in the EC's goal, a single flight represents only a portion of each passenger's journey. A full door-to-door journey may include multiple flights and also rely on other modes of transportation, such as rail, road and shipping. A delayed flight can disrupt each subsequent stage, leading to missed connections and hence whole-journey delays that are far greater than flight-level statistics might suggest. Flight cancellations can have a particularly large effect [2]. Currently, passengers on cancelled flights are typically offered a seat on a later flight by the same carrier. Depending on schedules and seat availability, some passengers may find a day or more added to their journey time. When major disruption occurs at an airport and many flights are cancelled, the number of passengers who need seats on later flights can far exceed the number of short-term seats available, leading to the potential for extremely long passenger-level delays. For example, in the US year-2000 data simulated by Bratu \& Barnhart [3] only 7\% of passengers are affected by disruption, but they suffer over half of the total passenger delay.

Not all disruptive events are predictable and there is no one single dominant cause, with weather, strikes, terrorism, system failures and volcanic ash, amongst others, all causing significant disruption in the last ten years [4]. For example, the eruption of the Icelandic volcano Eyjafjallajökul in 2010 and subsequent ash cloud caused Europe-wide disruption, including disruption to ground transport services such as Eurostar from large numbers of stranded air passengers switching modes [5]. Similarly, snow and ice in December 2010 resulted in the closure of a number of European airports, including London Heathrow and Paris Charles de Gaulle, and caused severe disruption at other airports, such as Toulouse, which had to accept large numbers of diverted flights at short notice [6].

There has been extensive research on network effects of disruption and disturbance propagation in the airspace $[7,8,9,10]$, the impact of airline scheduling of aircraft and crew [11] and the best recovery optimization schemes $[12,13,14,15,16]$. A general review of airline system disruption risk management research is given in Chung et al. [17]. Recently, there has been a shift towards passenger-centric rather than flight-centric metrics in air transportation disruption research, as reflected by the EC's 2050 goal $[3,18,19,20,21,22]$. In particular, Zhang 
and Hansen [23] developed a framework to reduce passenger disutility due to delay and missed connections via real-time intermodality specific to the United States transportation system. Real-time intermodality includes the substitution of flights by surface vehicle trips and, when the hub is part of a regional airport system, the use of inter-airport ground transport to enable diversion of flights to alternate hubs. Dray et al. [24] provide an analysis of the consequences of the Asiana crash on the multimodal transportation system in the San Francisco Bay Area, which was further explored by Marzuoli [25]. However, there is relatively little research looking at whether real-time intermodality would be feasible in Europe, even though many characteristics of European transport systems (e.g. widespread high-speed rail networks) suggest that it might be more suited to a European context than an American one. Although some airports, such as Frankfurt and Toulouse, do make use of ground transportation options to transport passengers affected by specific (primarily domestic) flight cancellations, this is not a commonly-used option at most airports[6]. Additionally, the increasing number of passengers travelling with smartphones opens up more options for implementation. This paper addresses this gap in the literature, using new data collected as part of the recent EC FP7 project Multimodal, Efficient Transportation in Airports and Collaborative Decision Making (MetaCDM; [26]). We propose that European airlines may be able to make significant savings on the cost of delay by increasing their use of alternative modes to get passengers to their final destinations in disrupted situations. To address this question, we model disruptive events at a range of European airports and their recovery periods, including the option for passengers to travel by alternative modes, and optimize for total airline cost of delay. This work also addresses a wider range of alternative transportation options than previous work in this area, including bus, train, alternative flight, alternative carrier and alternative airport options.

The rest of this paper is structured as follows: Section 2 discusses the feasibility of using alternative modes to help stranded passengers in situations where significant disruption occurs at an airport, both in a more general sense and by optimising for airline passenger delay-related costs. Section 3 discusses the results of the optimisation, including a sensitivity analysis of key uncertain parameters. Section 4 discusses how such a concept could be practically implemented. Finally, Section 5 draws conclusions.

\section{FEASIBILITY OF USING ALTERNATIVE MODES}

To be successful, any use of intermodality to alleviate aviation disruption should benefit all stakeholders. In particular, passengers should expect to see a sizeable reduction in delay at their final destination, and airlines a reduction in costs. We use these as the key metrics in assessing whether the concept is feasible. The two 
outcomes are somewhat aligned, because airline costs increase as passengers become more delayed and the airlines are required by EC regulations to provide food, hotel rooms, and/or ticket price refunds. However, airlines may choose to subject some passengers to longer delays if by doing do they can reduce their delay costs by a greater amount elsewhere, so the airline cost optimum does not necessarily reduce delays for all passengers.

To assess feasibility, we combine real-world data on existing European air and ground transport systems with a set of simulated disruptive events and optimise to find the lowest-cost solution for re-accommodating passengers. We also examine the impact on delay in each case. Additionally, any strategy to use other modes must also be feasible on a legal, safety, accessibility and systems compatibility basis amongst others, and must rely on systems which facilitate co-ordination and planning between the different stakeholders involved. These implementation-related issues are discussed in Section 4.

\subsection{Data}

We simulate disruptive events on a network including the top 50 European airports by passenger traffic in 2012 , as listed by ACI [27] ${ }^{\dagger \dagger}$. A comparison of the characteristics of existing air, rail and road options on routes between these airports in 2012 is shown in Figure 1. Data on air schedules, journey times and capacity provision by air are taken from OAG [28]. Year-2012 typical load factors by route use data from Eurostat [29]. Air ticket costs for legacy and low-cost carriers use European modelling from the AIM aviation systems model [30].

For rail, we use the ETISplus [31] database to get data on historical journey times, fare, frequency and number of changes required, supplemented by data from online journey planners [32]. Rail journeys with many changes are typically not feasible options for re-accommodating passengers, both in terms of journey time and logistical complexity. As shown in Figure 1, this rules out most long-distance rail substitution. Information on typical rail capacity and load factors is taken from Andersson et al. [33]. To generate schedules from frequency data, we assume an even distribution of rail services throughout the day, excluding the 1am-5am period.

\footnotetext{
† We include Turkish airports and exclude Russian ones, as this is the scope used by the reports on airport delay and disruption studied in the MetaCDM project. The 50 airports are, by ICAO code, LHR, CDG, FRA, AMS, MAD, IST, MUC, FCO, BCN, LGW, ORY, AYT, ZRH, CPH, PMI, VIE, OSL, DUS, MAN, ARN, DUB, BRU, MXP, TXL, STN, LIS, HEL, SAW, GVA, HAM, ATH, AGP, NCE, PRG, LPA, STR, LTN, WAW, ADB, CGN, ESB, LIN, EDI, BHX, BGY, ALC, TFS, BUD, LYS and MRS.
} 

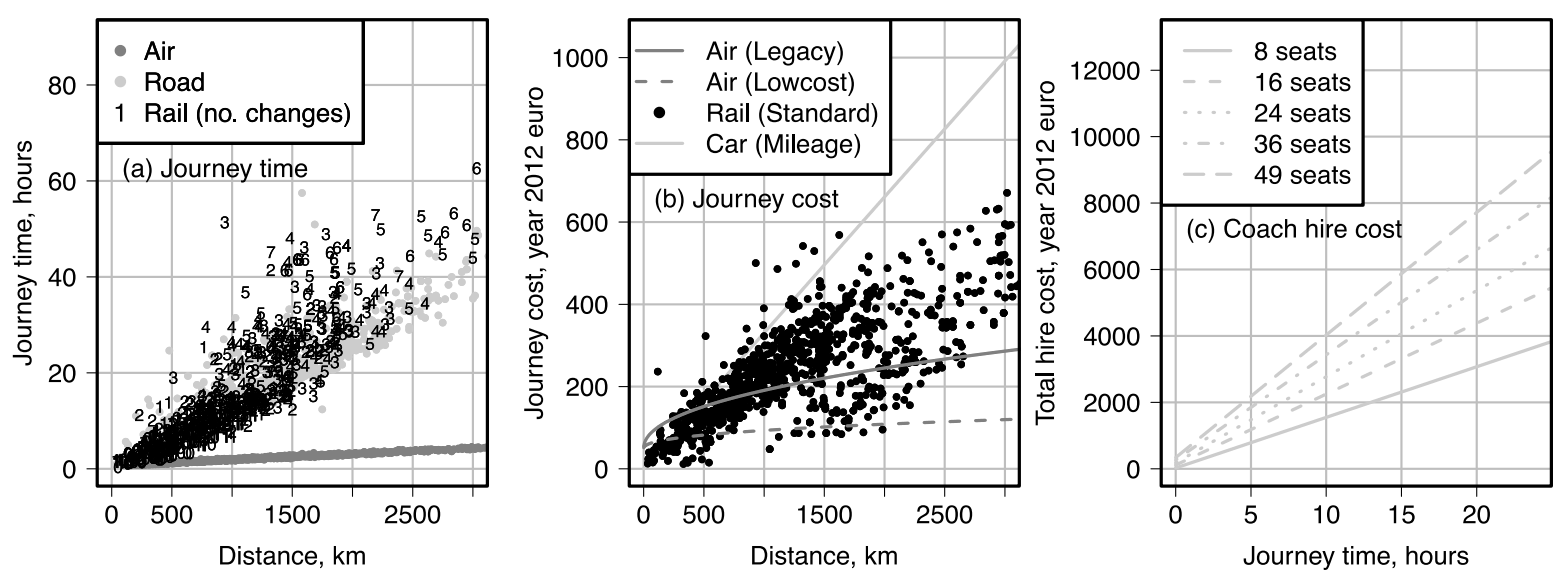

Figure 1. System journey time (a) and cost (b) data, and coach hire cost model (c).

For road, a number of options are possible; these include passengers hiring a car or driving their own vehicles, passengers relying on scheduled bus or coach services, and airlines chartering coaches or maintaining their own coach fleets. Car use, however, is relatively high cost. A typical car mileage rate from HMRC [34] is shown in Figure 1(b). Airlines using car-based re-accommodation options as an alternative to flights are likely to have to pay the costs accruing to the passenger, and these are significantly above costs for most air and rail services. Due to this and the likely complicated logistics of this option, we exclude cars in this analysis. Scheduled bus and coach services are also excluded, due to lack of suitable data. Instead, we concentrate on airline coach hire as the main road-based option, following [23].

In the near future, self-driving vehicles may also be available. In this case costs and likely usage may differ radically from the car options outlined above. For example, airlines could operate fleets of self-driving vehicles like long-distance taxis, optimising routing to deliver passengers directly to the doorstep of their final destination in as short a time as possible. The different possible use cases potentially make self-driving vehicles a feasible re-accommodation option to be explored in future analyses.

Undisrupted road journey time data by route is obtained from online journey planners [35, 36]. Road journey costs and available capacity are less easy to estimate. We model coach hire costs as having a fixed element (including fleet-related and administrative costs) and an element that is proportional to journey time (including fuel and driver costs). We carried out an informal survey of costs for coach hire between European airports $(\mathrm{N}=$ 41, covering 11 operators and airports in 13 countries, including long-distance journeys and those between airports serving the same city). To form a common basis with cost estimates for the other modes, we specified 
advance rather than last-minute costs; the impact of potential variation in costs due to last-minute requirements is then explored in the sensitivity study (Section 4.2). Based on this data, we model coach costs by size as a function $C B H / V_{z}=33.2+0.86 t$, where $C B H$ is the cost of hiring a coach, $z$ is the number of seats and $t$ is the (one-way) journey time $\left(\mathrm{R}^{2}=0.81\right)$, as shown in Figure $1(\mathrm{c})$. Due to confidentiality requirements attached to some of the coach cost quotations, survey data points are not shown in the figure. The total capacity of coaches to take passengers will depend on the stock of coaches that are available for short-notice hire in the local region. For Europe, data is available on the total stock of coaches by region [29]. However, the vast majority of these coaches will be in use or not available for hire. As coach stock is both important to model outcomes and uncertain, we include the proportion of these coaches that will be available for hire in the sensitivity study carried out in Section 3.2, below.

Road and rail journey times and costs include ferry segments where necessary to complete the journey. We assume schedules and journey times remain broadly similar over the 2010-2014 period, and that prices remain similar in real terms (all costs are quoted in year 2012 euros).

Initial analysis of this data [26] suggests that greater use of ground transportation is a feasible option for transporting stranded passengers, but that costs may be a concern. Although fewer than $3 \%$ of connections between the 50 airports can be substituted by airport-airport high-speed rail links, around $85 \%$ of connections can be substituted by city-city rail of some type. If including road and ferry connections, $96 \%$ of connections can be substituted by ground transport, and if additionally including the option of ground transport to an alternative airport, all of the connections can be substituted. For a time to next available flight from the original airport of 10 hours, over $50 \%$ of passengers could reach their destination faster using ground transportation. If the next available flight is 24 hours later, over $85 \%$ of passengers on links between the 50 -airport set could arrive sooner by ground transportation, although this may mean travelling overnight. However, costs for ground transportation are often higher than those for the comparable flight (Figure 1). In addition, there are complicated relationships between available schedules and seats, load factor, the number of passengers needing reaccommodation, re-accommodation costs and passenger delay costs that will determine whether it is beneficial to send no, some or all stranded passengers via ground transportation. Because of these complexities, we approach the problem as an optimisation exercise, using the above data from the 50-airport set described at the start of this section. 


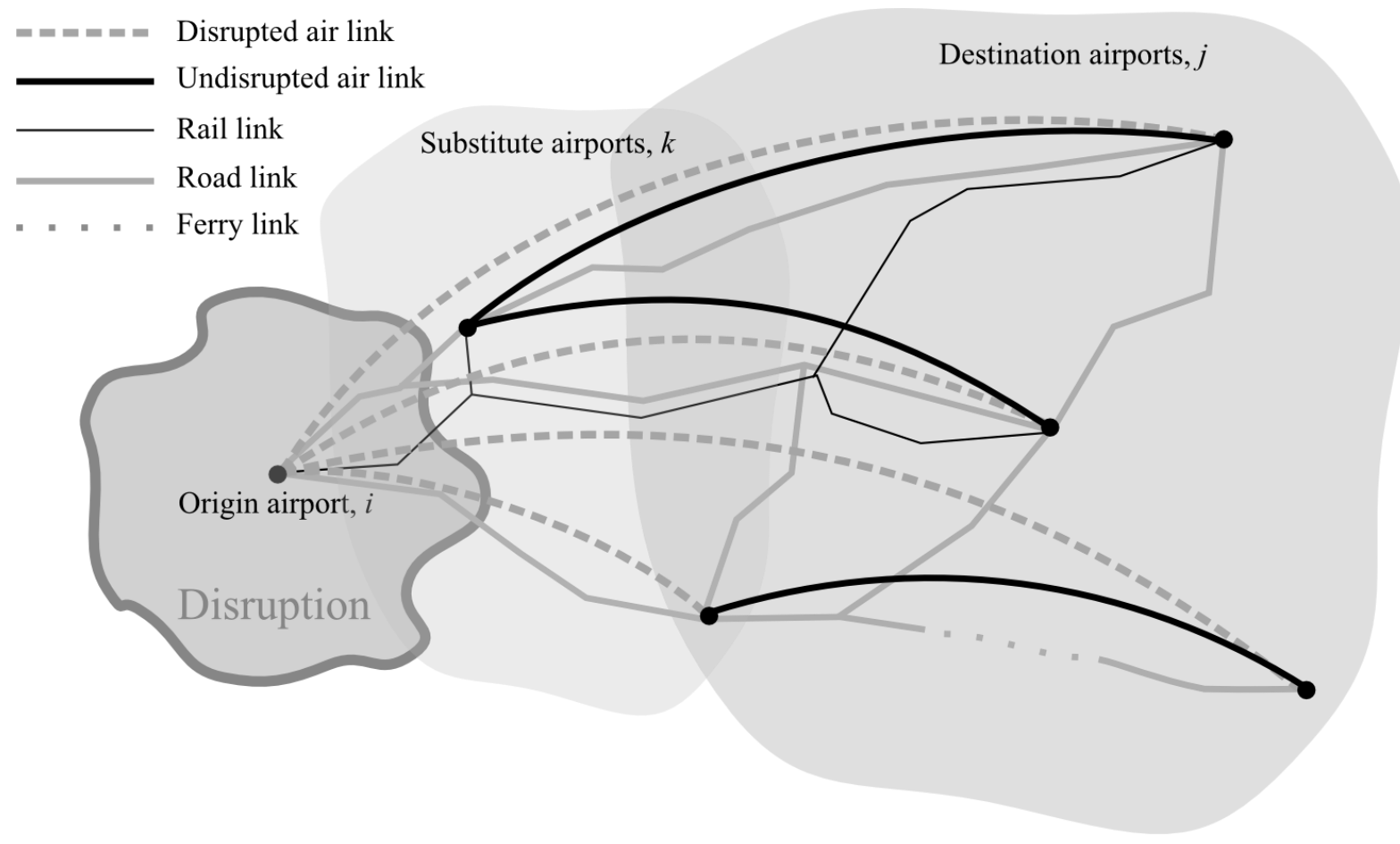

Figure 2. Representation of links between a sample set of airports.

\subsection{Optimisation}

We assume a disruption occurs at origin airport $i$, resulting in some number of flights to destination airports $j$ being cancelled (Figure 2). Each cancelled flight $c$ carries $N_{i j c}$ passengers. Of these passengers, some proportion $P_{R O}$ will choose, in accordance with their rights, to be reimbursed and either not fly or be returned to their point of origin (depending on whether they are at their point of origin or not). We assume half of reimbursed passengers will still require re-accommodation, i.e. a total of $\left(1-P_{R o} / 2\right) N_{i j c}$ passengers per cancelled flight are stranded and need to be transported to destination airport $j$ by some alternative method. We do not model the potential need to catch connecting flights at airport $j$ in the optimisation, due to a lack of passenger itinerary data. The proportion of connecting passengers varies by destination airport and is typically lower at non-hub airports and those used by low-cost carriers. For example, around 27\% of passengers at London Heathrow are connecting passengers, but less than 3\% at London Gatwick [37]. Our model will be more accurate for airports that link primarily to these airport types.

Following the period of disruption, we assume a full flight schedule of operating flights $(f)$ resumes. This represents a simplified view of real disruptive events, which can sometimes have variable numbers of flights operating during/after the main disruption period, and where airlines may choose which flights to delay and which flights to cancel based in part on the difficulty of re-accommodating passengers [38]. These flights have 
$S_{i j f}$ spare seats available which can be used to accommodate stranded passengers. In addition, trains $T$ may be operating between the origin and destination cities, with $S_{i j T}$ spare seats, and some number of coaches of size $z$ each with $S_{z}$ seats, $N B_{i z}$, may be available for short-notice hire. Flights to destinations $j$ may be operating from other nearby airports $k$, with $S_{k j f}$ seats available.

We assume five possible cases for re-accommodating passengers: a later flight from the same airport to the destination city (by the same airline, an alliance partner or a non-aligned airline); train from the origin to the destination city; coach of size $z$ from the origin to the destination city; train to an alternative airport followed by a flight to the final destination; and coach of size $z$ to an alternative airport $k$ followed by a flight to the final destination. These are not the only possible options. For example, we exclude the cases where passengers take a flight to an alternative airport followed by ground transport to their final destination, and more complex composite journeys such as coach + train, coach + flight + coach, etc. These are omitted to limit the size of the optimization and, in the case of flight + ground transport, because of the likelihood of the initial flight in this case also being affected by the disruption and recovery period.

Not all airports can offer all options. For example, airports on islands have limited ground transport alternatives. Although the choice of alternative should ideally be a passenger one, the choice of which options to offer will be set by the airlines and in many cases the interests of the passenger and the airline will be aligned. In this paper we assume the choice is set by airlines rather than passengers, and that airlines aim to minimise their costs. We explore the system cost optimum rather that the cost optimums for each individual airline. Because airline costs are dependent on the length of time passengers are delayed for, the optimum airline cost solution should also reduce passenger delay. For simplicity, we concentrate on outgoing passengers. We assume a similar process takes place for passengers whose incoming flights to airport $i$ are cancelled, but do not model this in detail.

The cost of delivering passengers to their final destination varies depending on the method used. For flights, we assume zero extra cost if the carrier is either the passenger's original carrier or an alliance partner of that carrier. For other airlines, and for train services, we assume a cost similar to a typical passenger fare as estimated by flight distance in Figure 1. Coach hire costs are estimated using the survey-based relationships estimated in Section 2.1 (Figure 1). We assume 8- and 16-seater coaches will mainly be used only for inter-airport transfers as this was the primary use case given when carrying out our coach costs survey. This is done by limiting the model journey length where they can be used to under 2 hours. It should be noted that these assumptions ignore two factors that may work in opposing directions. First, the fares and/or hire costs for alternative transport could 
be assumed to be walk-up fares, which are typically higher. Second, it is likely in this situation that the airlines would have pre-arranged deals with other transport providers, as is currently the case with hotel providers, to lower prices when dealing with disruption. These issues are explored in the sensitivity study (Section 3.2).

Table 1. Airline hard cost assumptions by length of delay, adapted from [20].

\begin{tabular}{|c|c|c|c|c|}
\hline & \multirow[t]{2}{*}{ Provision } & \multicolumn{3}{|c|}{$\begin{array}{l}\text { Cost (year } 2012 \text { euro) by } \\
\text { scenario, } 2 \text { s.f. }\end{array}$} \\
\hline & & Low & Base & High \\
\hline $1.5 \leq \mathrm{t}<2$ & Refreshment & 0 & 1.8 & 2.1 \\
\hline $2 \leq \mathrm{t}<3$ & Refreshment and tax-free-voucher & 4.9 & 8.1 & 9.9 \\
\hline $3 \leq \mathrm{t}<5$ & $\begin{array}{l}\text { Refreshment, tax-free voucher, meal voucher and FFP } \\
\text { miles }\end{array}$ & 13 & 20 & 24 \\
\hline $\mathrm{t} \geq 5$, not & Refreshment, tax-free voucher, meal voucher, FFP & 14 & 22 & 27 \\
\hline overnight & miles and ticket discount voucher & & & \\
\hline Overnight & As above plus hotel accommodation & 54 & 87 & 105 \\
\hline
\end{tabular}

The total cost to the airline of a stranded passenger includes reimbursement and potential re-accommodation costs, as discussed above, and also passenger delay costs to the airline. The latter can be divided into hard costs (i.e. the cost of providing food, hotel rooms and other services for stranded passengers) and soft costs (i.e. the costs incurred due to disgruntled passengers switching to other airlines in future). These costs are modelled as functions of the length of individual passenger delay, following Cook et al. [20]. Hard cost assumptions are shown in Table 1, are additive, and are assumed to repeat daily starting from noon for delays of over a day. Soft cost assumptions are shown in Table 2; as in [20], we assume that soft cost saturates at delays of 300 minutes (i.e. passengers are already as annoyed as they are going to get when the delay gets this large).

Table 2. Airline soft cost assumptions by length of delay, adapted from [20].

\begin{tabular}{|c|c|c|c|c|c|c|c|c|c|}
\hline Delay (minutes) & $\overline{5}$ & 15 & 30 & $\overline{60}$ & 90 & 120 & 180 & 240 & 300 \\
\hline Low Cost Scenario, $€_{2012}$ per & 0.01 & 0.02 & 0.07 & 0.20 & 0.26 & 0.28 & 0.28 & 0.28 & 0.28 \\
\hline \multicolumn{10}{|l|}{ passenger minute } \\
\hline Base Cost Scenario, $€_{2012}$ per & 0.02 & 0.09 & 0.26 & 0.73 & 0.96 & 1.01 & 1.02 & 1.02 & 1.02 \\
\hline
\end{tabular}


passenger minute

\begin{tabular}{|c|c|c|c|c|c|c|c|c|}
\hline High Cost Scenario, $€_{2012}$ per & 0.03 & 0.11 & 0.29 & 0.81 & 1.06 & 1.11 & 1.13 & 1.13 \\
\hline
\end{tabular}

These cost functions include high, low and base scenarios reflecting uncertainty about the exact circumstances of each situation - for example, local hotel costs and occupancy, passenger preferences on each flight and the arrangements individual airlines have made to look after passengers. In our base case, we use the central projections given for hard and soft costs, but the other values are explored in the sensitivity study in Section 3.2.

To estimate journey times and the delay at final destination associated with each method, we use the real-world schedule and travel time data discussed in Section 2.1, in combination with estimated buffer times accounting for logistical delays. We assume at least one and a half hours is needed for passengers to be informed of the cancellation and to travel to the start site of the alternative journey (for example, a train station); at least four hours is needed for airlines to provide coaches ready for passengers [23]; and at least two hours is needed between passengers arriving at an alternative airport and their flight departing. These factors are highly uncertain and will in reality depend on a number of difficult-to-model factors. We explore using a range of different values in the sensitivity study (Section 3.2).

Finally, the capacity of each mode to carry stranded passengers needs to be estimated. For flights and train trips we use actual and estimated schedule and load factor data, as discussed in Section 2.1. Short-notice coach availability is highly uncertain. We assume the total number of coaches available for hire is a given fraction $P_{C A}$ of the total regional coach stock [29], and is evenly distributed between five size classes with 8, 16, 24, 36 and 49 seats respectively. For the baseline runs a value of 2 percent for $P_{C A}$ is assumed, and this is also explored in the sensitivity study. We do not model the case where more passengers are carried than seats available, as this is only feasible for modes which can take standing passengers (i.e. some train-only and some coach-only trips) and carries the risk of decreasing both passenger and ground transport operator buy-in.

Optimizing in this way also makes a number of effective assumptions about passenger behaviour. We assume that passengers will be able to keep in contact with the airline and other stakeholders at all times. We also assume that all passengers will understand and be able and willing to follow the instructions they are given and will agree with the airline's choice about which option they should take. Similarly, we assume all transport stakeholders will be contactable at all times, including at night. In real-world situations passenger behaviour is 
likely to be more diverse and complex, and any real-world system will need to be adaptable to deal with this. Similarly, the optimization produces the lowest-cost solution available given full information about how long the disruption will last, which flights are cancelled across all airlines, and how many passengers need to be reaccommodated. In a real-world disrupted situation, much of this information will not be initially available to airlines and any optimization process will need to be adaptable to incomplete information that is subject to frequent updates. These constraints will reduce the overall benefits available, i.e. our results represent an upper bound on what is achievable.

A full mathematical description of the optimization is given in Appendix A. The optimisation was programmed in R using the rcplex interface [39] to IBM's CPLEX for solution.

\section{RESULTS AND DISCUSSION}

\subsection{Main Outcomes}

For our base scenario, we assume a hypothetical block of cancellations at London Heathrow airport, beginning at 8 am on a Wednesday in May 2012. Historical disruptions at Heathrow and their recovery were extensively studied by the MetaCDM project $[6,26]$, on which this work is based. We consider only flights within the 50airport set, and assume that all flights within a given time period are cancelled, and all flights afterwards operate normally; ground transportation is assumed to be unaffected by the source of the disruption. Figure 3 compares two cases. In the reference case, passengers on flights that are cancelled are re-accommodated only on flights by the same carrier and/or their alliance partners. In the alternative case ('plus alt modes'), they may also be transported to their destination by alternative modes, as discussed in the previous section. We also assume that airlines are able to match passengers to the available alternatives such that, if the optimal solution for a flight involves $20 \%$ of passengers taking a train, there will be at least $20 \%$ of passengers who are able and willing to take this option without extra incentives from the airline.

Figure 3(a) shows the impact on total passenger delay-related costs (including re-accommodation costs) to airlines of providing alternative transport options. Total costs are similar in both cases, reflecting both a relatively small percentage of passengers who are switched to alternative modes and a relatively small average benefit per passenger. Where alternatives are available, total costs are reduced by around $15 \%$ for cancellation periods of under an hour, decreasing to around $2 \%$ for cancellation periods of around 5 hours, and rising again to a $6 \%$ reduction for a 10 -hour cancellation period. However, absolute costs for short cancellation period 
lengths are relatively low, so greater total savings are still made at higher cancellation period lengths (absolute cost savings are 6.5, 6.9 and $47.0 \mathrm{k} €(2012)$ for 1, 5 and 10-hour closure periods respectively). These figures arise from the different opportunities available with different cancellation period lengths. For short $(<1 \mathrm{hr})$ cancellation periods, around $30 \%$ of passengers take advantage of alternative options. Most of these passengers are put on later flights by non-aligned carriers rather than taking ground transportation, as shown in Figure 3(c). For longer cancellation periods, opportunities to put passengers on later flights by non-aligned carriers decrease, as those carriers are now also suffering disruption. For cancellation periods of around 3-4 hours, only around $15 \%$ of passengers take alternative options. These passengers are distributed relatively evenly between different alternative modes (Figure 3(c)).

(a) Passenger delay costs

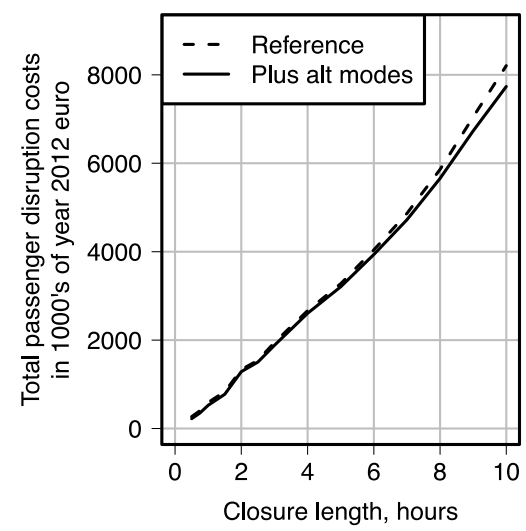

(b) Mean passenger delay

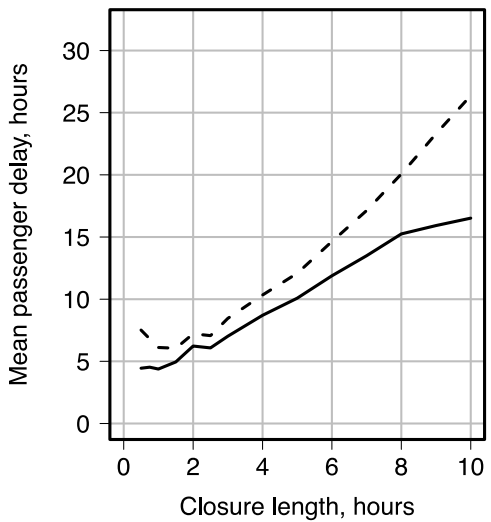

(c) Use of alternative modes

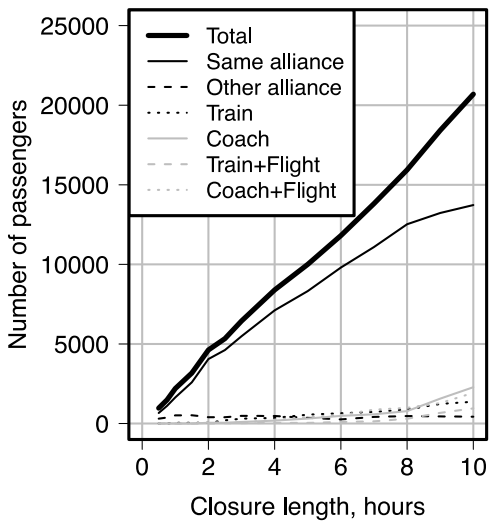

Figure 3. Cost (a), mean passenger delay (b) and alternative mode use (c) for simulated disruptive events at London Heathrow.

As the cancellation period increases towards 10 hours, the number of stranded passengers increases and the number of spare seats available for those passengers on later flights decreases. At the same time, airlines have an incentive to get passengers to their destination before regulations specify that they need to provide overnight hotel accommodation, which increases costs significantly. These incentives increase the number of passengers using alternatives once again. For a 10-hour cancellation period, 34\% of stranded passengers use alternatives, primarily ground transport options.

The impact on mean passenger delay (Figure 3(b)) is greater than the impact on costs. Mean passenger delay for short $(<1 \mathrm{hr})$ and very long $(>8 \mathrm{hr})$ cancellation periods is reduced by $30-40 \%$. For mid-range cancellation periods (3-5 hours), mean delay reduces by around 15\% despite the much smaller benefits in terms of cost. Although underlying delay distributions are similar in both cases, a larger number of passengers experience very 
short delays ( $<5$ hours) in the alternative case, and fewer passengers experience very long delays ( $>1$ day).

This reflects two specific pressures on delay cost. Soft costs of delay are assumed to saturate after 5 hours, but increase strongly over delayed time for shorter delays. Therefore there is significant benefit for airlines in providing alternative methods of travel if they can reduce passenger delay below this threshold. Second, as noted above, hard costs of delay increase strongly if the airline has to provide overnight accommodation.

Therefore there is an incentive for airlines to provide alternative methods of transportation if they can get the passenger to their destination before an overnight stay is required.

Many of the factors affecting outcomes may be specific to London Heathrow: for example, passengers taking ground transport options to continental European airports will have time and cost added to their journeys by the necessity of crossing the Channel, and Heathrow is part of a large multi-airport system that offers multiple flight alternatives. As we use air and ground transport data for all links between airports in our fifty-airport set, and full air schedules for all airports, we can also apply the model to similar disruption at other airports in the set. In Figure 4 we show the impact of closure events at other major European airports, using the same base parameters as for the Heathrow simulation (8am start, etc.).

(a) Passenger delay cost savings

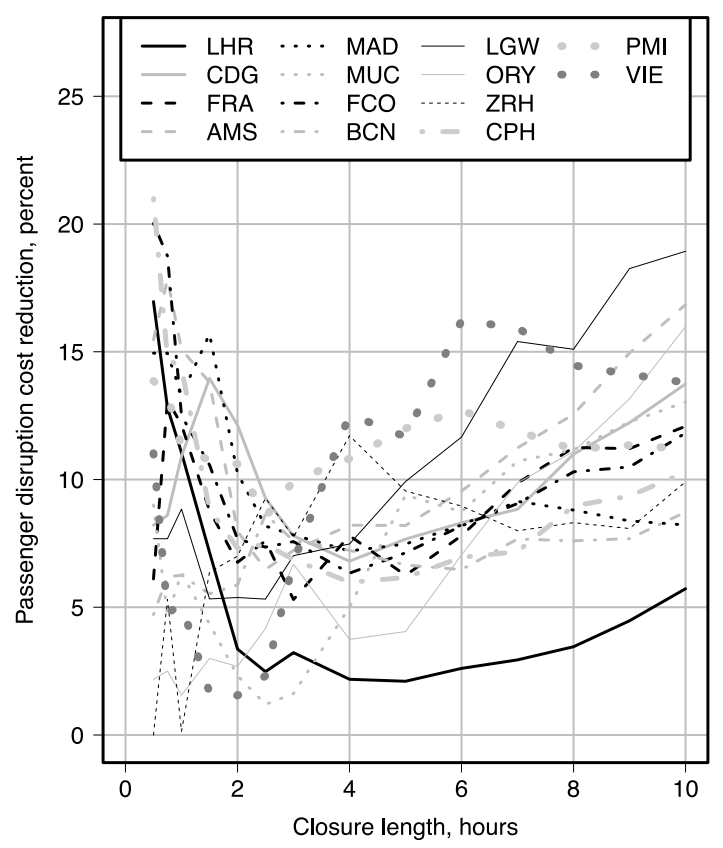

(b) Passengers using alternatives

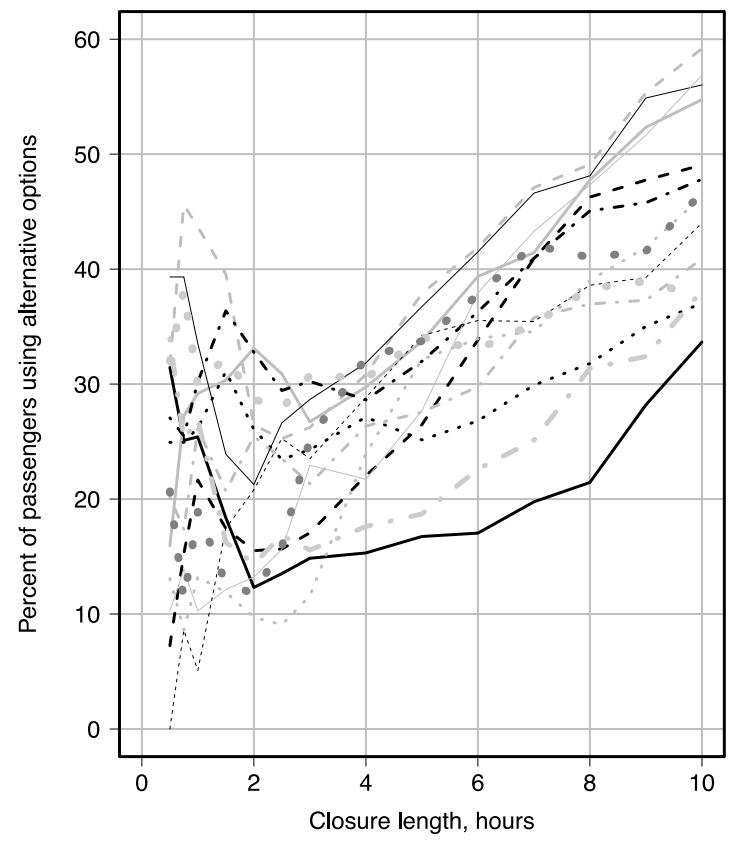

Figure 4. Reduction in disruption cost (a) and proportion of passengers using alternatives (b) for major European airports.

Several broad features remain similar between different airports. These include an initial peak in passengers using alternatives for short delays, caused by the relatively high availability of alternative flights from non- 
aligned carriers; a minimum in cost reduction and passengers taking alternatives around closure lengths of 2-6 hours; and a subsequent increase in both measures for longer delays as fewer alternative flight options from aligned carriers are available. However, the absolute level of benefits available from allowing alternative modes varies by airport, including up to a $20 \%$ reduction in passenger disruption-related costs in situations where ground transport links are particularly favourable. The need for most ground options to cross the Channel means that relatively few alternatives are used at Heathrow (LHR) in comparison to airports in Continental Europe. Similarly, Madrid Barajas (MAD) experiences relatively low benefits as it is constrained by its relatively distant location. Secondary airports, such as London Gatwick (LGW) and Paris Orly (ORY), show high benefits because they can use the larger primary airport as an alternative. The greatest benefits are seen by airports in North-Western Continental Europe, which has high population and airport density, few barriers for ground transportation and widespread high speed ground transport networks. For example, Paris (CDG and ORY) and Amsterdam (AMS) experience particularly high benefits, including up to $60 \%$ of passengers using alternatives for the case of a 10-hour disruption period, and a $60-70 \%$ reduction in mean passenger delay, because of their high-speed rail network links.

Another factor affecting the available benefits is the typical load factor of flights from the airport. Flights operating at high load factors (often leisure flights to holiday destinations) have reduced spare capacity to accommodate stranded passengers from previous flights and can therefore benefit more from the use of alternatives, where these are available. This means that, for example, Palma de Mallorca (PMI) experiences mid-range benefits even though its island location means that ground transport journeys need to include a ferry trip.

\subsection{Sensitivity study}

Many of the values for key parameters in the model are highly uncertain. These parameters include the number of coaches available at short notice, the time needed to get coaches to a suitable pick-up point, and the costs associated with passenger delay. In addition, some of these parameters are strongly dependent on the amount and type of preparation for disruption undertaken by the airport and airlines (for example, if airlines have prenegotiated ticket prices with other operators). Others will be dependent on the current or future infrastructure available at the airport. The time needed to get to a suitably-connected train station will be much lower for airports which are directly linked into high-speed rail networks than it will for airports which are on remote islands. Other parameters, such as the amount of buffer time required when travelling to an alternative airport, 
may depend on the nature of the disruptive event and whether ground transport modes are also affected. To test the impact on variations in these parameters on overall outcomes, we carry out a sensitivity study. As a baseline, we use the model run for a 10-hour closure at London Heathrow beginning at 8 am with all alternative options available. Outcomes are shown in Figure 5, and are discussed below. Panel (a) shows delay and total cost outcomes, panel (b) the number of passengers using alternative flights from the same or another airline, panel (c) the number of passengers re-accommodated by coach or train, and (d) the number of passengers reaccommodated using ground transport to an alternative airport, followed by a flight to their destination.
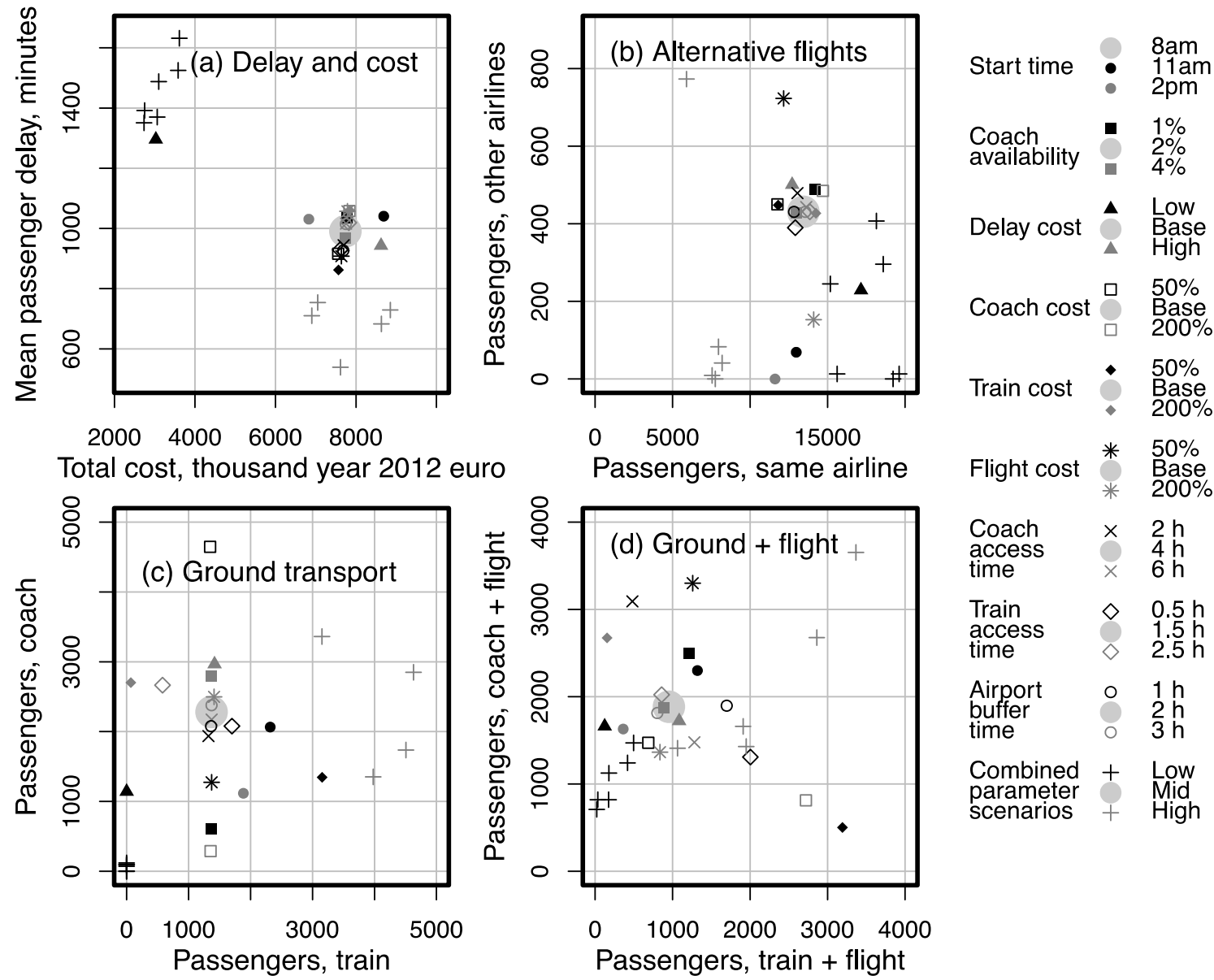

$$
\begin{array}{lll}
\text { access } & \times & 4 \mathrm{~h} \\
\text { time } & \times & 6 \mathrm{~h} \\
\text { Train } & \diamond & 0.5 \mathrm{~h} \\
\text { access } & \begin{array}{l}
1.5 \mathrm{~h} \\
2.5 \mathrm{~h}
\end{array} \\
\text { time } & \diamond & \\
\text { Airport } & \circ & 1 \mathrm{~h} \\
\text { buffer } & & 2 \mathrm{~h} \\
\text { time } & \circ & 3 \mathrm{~h} \\
\text { Combined } & + & \text { Low } \\
\text { parameter } & + & \text { Mid } \\
\text { scenarios } & + & \text { High }
\end{array}
$$

Figure 5. Sensitivity of model outcomes to key input parameters, for a 10-hour closure at London

\section{Heathrow airport.}

To test the model sensitivity, we first vary each uncertain parameter individually, keeping all the other parameters at base scenario values. The values used in each case are shown on the right-hand side of Figure 5 and are discussed individually below. However, this does not explore the full range of system variability as there may be interactions between different parameters. Therefore we also run a range of combined parameter scenarios. In the 'Low' scenarios we choose a set of input parameters which reflect a very difficult situation for 
ground transport use. For example, the low delay cost scenario is used, the cost of train and coach travel is assumed to be twice what it is in the base runs, and access times are assumed to take the high values from the individual parameter runs in each case. We run these scenarios for a range of disruption times of day and alternative flight cost. We do the same for a set of 'High' scenarios in which input parameters reflect easy situations for ground transport use: high delay cost, train and coach travel cost at $50 \%$ of its base scenario value, and access times which use the low values from the individual parameter runs.

The greatest source of variability is the delay cost scenario. We use delay costs from [20]; these scenarios are not symmetric around the base case, with the low-cost scenario having significantly lower costs than the other two (Table 1, Table 2). For example, the total cost of 24 hours' passenger delay is $12 \%$ higher in the high-cost scenario than in the base scenario, but $62 \%$ lower in the low-cost scenario than in the base scenario. The lowcost scenario may be appropriate to airlines with a business model which provides only the minimum amount of assistance necessary to comply with regulation. Outcomes for the high and base delay cost cases (filled triangles in Figure 5) are relatively similar, but the low delay cost case has a significant impact on model outcomes, with only $15 \%$ of passengers using alternatives (compared to $34 \%$ in the base delay cost case), and mean passenger delay at final destination of 21.6 as opposed to 16.5 hours. The combined-parameter runs which use the low-cost scenario for costs (black plus signs in Figure 5) have similar outcomes.

Filled circles show the impact of closure start time. As discussed above, the extra cost of accommodating passengers overnight acts as an increased incentive to provide alternatives that will enable passengers to reach their final destination before a hotel stay is needed. Later closure starts mean more flights are cancelled later in the day, increasing the proportion of passengers who use alternatives. However, the number of available options to do this decreases later in the day. For later start-of-closure times the end of the closure period also runs into the night-time curfew period when there are no or few flights to cancel, reducing the total number of passengers stranded. Both of these factors lead to a decreasing absolute number of passengers re-accommodated by current methods (i.e. later flight by the same airline) as the closure start time gets later. These outcomes are also reflected in the combined-parameter scenarios for different closure start times.

Filled squares show the impact of the coach availability parameter, $P_{B A}$, comparing the situations where $1 \%, 2 \%$ and $4 \%$ of wider regional coach stocks may be available for short-notice hire (for Heathrow, this corresponds roughly to 25,50 and 100 coaches of each size class respectively). In the $1 \%$ case, nearly all coach sizes reach this use constraint, whereas the $4 \%$ constraint effectively represents unconstrained coach use. Outcomes in 
terms of total delay cost show relatively little impact; the main result of reducing coach availability is to shift passengers on mid-length routes with poor train connections from coach substitution to waiting for a later flight.

Empty squares, filled diamonds and stars show the impact of changing the costs of coach hire and train and flight tickets respectively. In the high cost case, estimated costs are doubled, to simulate a situation where alternative operators take advantage of the situation by charging premium rates. In the low-cost case, estimated costs are halved; such a situation might arise if agreements have been made beforehand between operators. These cases make little difference to overall delay and cost profiles but alter the proportion of passengers taking each mode. For example, the difference in overall airline cost of passenger delay between the high and low coach cost scenarios is $3.7 \%$, even though the cost of hiring a coach differs by a factor of four.

Crosses, empty diamonds and empty circles show the impact of changing buffer times: the time required for short-notice coach hire, the time it might take passengers to access a railway station with suitable services, and the extra buffer time needed over the ground transport journey time (accounting for ground transport delays, check-in, security, etc.) to take a flight from an alternative airport. Low buffer times might be needed if passengers start their journey from an airport that is already connected to long-distance train services; the airline already owns a fleet of coaches it can use; or no delays are anticipated on the way to or at the alternative airport. High buffer times reflect situations where either the base case assumptions are overly optimistic, or ground transport modes are also moderately disrupted. However, as with the cost scenarios discussed above, the overall impact on total passenger delay cost and mean passenger delay is small. Instead, the main impact is on the balance of modes used. This is partly a function of the high degree of redundancy in alternatives available from Heathrow. Nearly all flights have multiple feasible options for ground transport substitution which can be used if the cost or time for another option increases. Airports in more constrained situations, such as those on islands, may experience larger impacts here.

The combined-parameter scenarios (plus signs in Figure 5) largely reflect the dominant impact of the delay cost scenario. For the 'Low' combined-parameter runs, which have low delay cost, high cost of alternative modes, and long wait periods, use of alternative modes is minimal, accounting for only $5-12 \%$ of passengers. This is similar to the case with low delay cost in which all other parameters are kept at base values. For the 'High' combined-parameter runs, which have high delay cost, low cost of ground transport, and short wait periods, use of alternative modes is high, at between $52-71 \%$ of passengers (total usage depends on the time of day and the scenario used for cost of alternative flights, which we vary in the combined-parameter runs). In the case where 
alternative flights also are at the low end of the cost range used, mean passenger delay is reduced to under nine hours from over sixteen hours in the base case.

As discussed in Section 3.1, the proportional amount of cost savings also depends on the length of the closure period, with the highest percentage decreases in passenger delay-related costs for short ( $<1 \mathrm{hr})$ and long $(>8 \mathrm{hr})$ closure periods. This variability arises due to the differing availability of alternative flights for different closure lengths, and may mean that shorter closure periods are differently affected by changes in the uncertain parameters. However, the model response to changes in uncertain parameters is similar for shorter closure periods, with three exceptions. First, closure start time has a smaller impact for shorter closure periods as less recovery time is needed, reducing the number of passengers who require overnight accommodation. Second, fewer coaches are used for shorter closure periods so the impact of the coach availability parameter is reduced. Third, for short closure periods ( $\sim$ hour) where there is relatively little use of ground modes, sensitivity to ground mode cost, availability and access time is significantly reduced.

\section{IMPLEMENTATION}

Our analysis demonstrates that the use of alternative mode rerouting may help to achieve reductions in passenger delays, airport overcrowding and other negative impacts of aviation system disruption. However, there are a number of important hurdles that would need to be overcome to practically implement this concept. These include barriers to information sharing (such as incompatible systems, reservations about collaborating with competitor organisations and passenger privacy concerns), liability issues and passenger buy-in. Airlines, airports and other stakeholders will need to work closely together, co-ordinating their response, and will need systems in place to help them do this. Significant planning will need to be in place before any disruptive event to ensure that resources can be mobilised at short notice, most notably between airlines and coach providers in the case that coach provision is envisaged. In this section, we discuss what a practical implementation strategy might look like. Similar problems relating to the streamlining of airport operations with a much smaller number of stakeholders have been successfully addressed by Collaborative Decision Making (CDM) concepts. The EC FP7 project MetaCDM [6, 26] explored how these concepts could be extended to passenger door-to-door journeys, using the successful and widely-used Airport CDM (A-CDM) standard [40] as a formal basis. In particular, increasing smartphone availability (for example, over three quarters of air passengers travelled with a smartphone in 2013; [41]) allows passengers to straightforwardly participate in the information exchange process in a way that has not previously been possible. 


\subsection{A-CDM}

A-CDM monitors the calculation and reachability of key milestones in an aircraft journey, such as the Target Start-Up Approval Time (TSAT) and Target Take-Off Time (TTOT) of an aircraft departure. The main objective is to check if a regulated flight is able to depart within its Air Traffic Flow Management (ATFM) slot and, if not, to adjust departure sequencing so that overall delay is minimized.

This is accomplished via six functional groups [40]: information sharing between airlines and the airport; a collaborative turnaround process in which milestones for different stages of flight readiness for departure are defined and monitored; calculation of the time taken between these milestones (variable taxi time calculation); collaborative management of flight updates providing frequent updates on progress towards milestones; collaborative pre-departure sequencing in which the most efficient order for aircraft to depart from their parking positions is calculated, taking into account operational constraints and airline preferences; and CDM in adverse conditions, where A-CDM partners anticipate and collaboratively manage periods when airport capacity is reduced due to adverse conditions. Eurocontrol [42] estimate that the 17 European A-CDM airports have seen an average $10 \%$ reduction in ATFM delay minutes and $7 \%$ reduction in taxi time due to their adoption of ACDM.

\subsection{P-CDM}

Using the A-CDM functional groups as a starting point, it is possible to define a Passenger-CDM (P-CDM) concept extending these ideas to the movement of passengers rather than aircraft [26]. In this concept, the passenger participates in the information exchange process, providing opt-in information about their progress towards key journey milestones such as boarding each flight stage in their journey. Airlines and other stakeholders in turn provide information about delays, likely process times and alternative transportation modes in case of disruption. As with A-CDM, information from all partners passes through a central P-CDM system which handles data sharing and confidentiality issues. Passenger interaction with this system would optimally be via a smartphone application, but a more limited version could be operated via email or SMS. Implementing such a system would be significantly more challenging than A-CDM, as there are many more stakeholders and because the stakeholders include individual passengers, each with their own preferences and requirements. 
As with A-CDM, any concept looking to streamline the movement of passengers in crisis situations must also be operational and provide benefits under non-crisis conditions, or it is unlikely to be adopted. Therefore we define both non-disrupted and disrupted modes of operation.

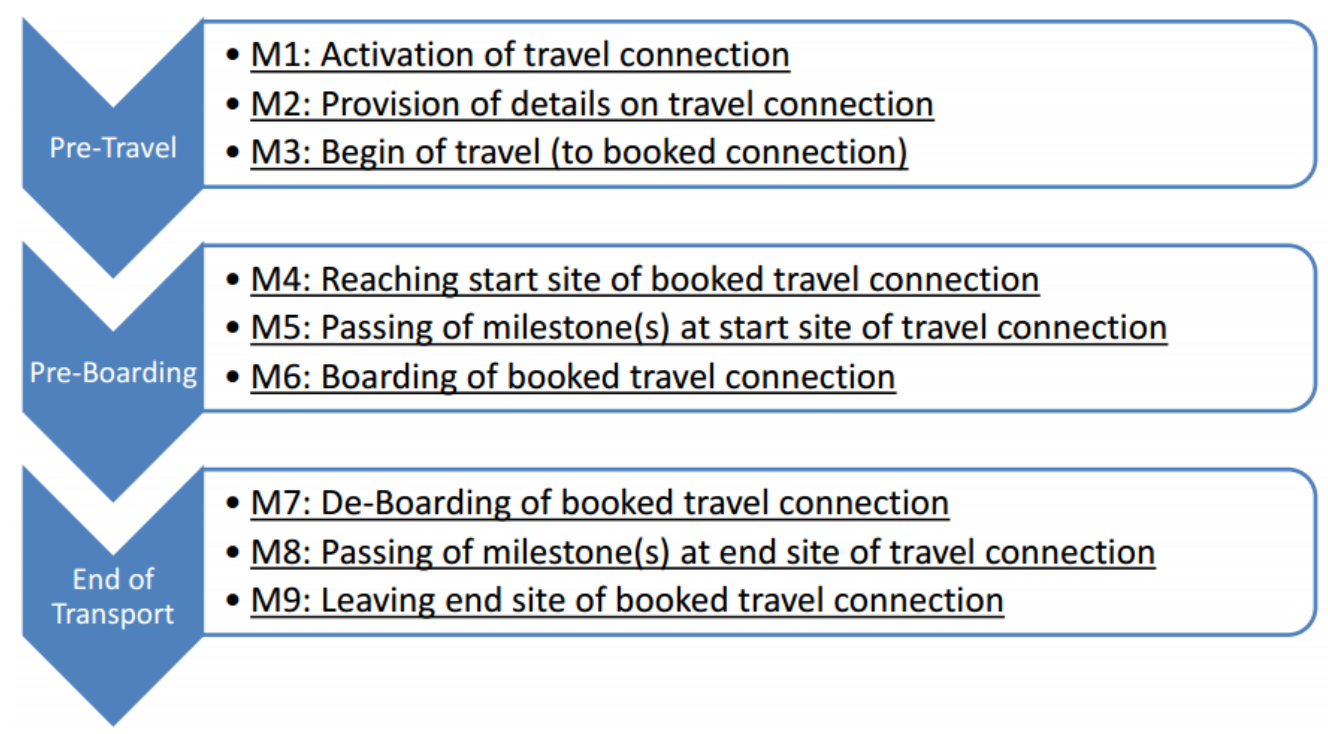

Figure 6: P-CDM Milestones (source: Laplace et al. (2014b))

\subsubsection{Operation in non-disrupted conditions}

Under non-disrupted conditions P-CDM would operate similarly to A-CDM but with a passenger door-to-door journey focus: for example, instead of using information exchange to assess whether a flight is able to depart within its ATFM, the ability of passengers to make key connections (such as boarding a flight) could be assessed. However, the fact that passengers are not aircraft (for example, the passenger at home is not subject to a controller) leads to some differences. A-CDM includes primarily airlines and airports, whereas passengers and ground transport operators would also need to be included in the P-CDM information sharing process to cover the whole journey. This introduces a much wider range of stakeholder requirements and complexity. The information exchange could cover customer needs, planned and estimated times at milestones, dynamic estimates of likely processing times (e.g. queueing time at security), target times from passengers at milestones, and potentially GPS position data.

Figure 6 shows how the passenger journey could be represented in terms of milestones for a non-disrupted journey. Milestones in the Pre-Travel phase go from the booking of the full door-to-door itinerary, through the sending of provisional itinerary details, to the start of the passenger journey. For example, a passenger might 
book a flight and ground transport connections (such a train to the initial airport and a bus from the destination airport to the final destination) via a P-CDM system. After the booking is made, the passenger receives all the details (bus, train and air travel schedules). These details can be updated if necessary. For example, if there are train delays then the passenger could be alerted and given the option of taking an earlier train to be sure of making their flight in time.

The Pre-Boarding phase includes all journey milestones up to the boarding of the aircraft. These could include arriving at the train station, boarding the train, arrival at the airport, baggage drop and security check. The End of Transport phase includes all milestones from the aircraft de-boarding to arrival at the final destination (such as a hotel). Example milestones here could be border control and boarding the bus to the final destination.

Throughout the journey, information is exchanged between the passenger and travel providers, with the aim of keeping the passenger updated on any factors that may affect their journey and the transport operators updated on passenger location. This allows airports to better plan staffing and resource use, and airlines to have better information about whether missing passengers will be able to make the flight. The overall aims of P-CDM under non-disrupted conditions are to reduce passenger journey and waiting times where possible and to reduce uncertainty for operators.

Within this process, different operators would be responsible for providing information on different target times and/or travel times between milestones. When a transport connection is booked, the provider will define a target time for boarding; any given passenger journey may include one or more travel connections from one or more service providers. Other milestones are defined by P-CDM to aid the passenger in meeting these target times (for example, a suggested time to leave home taking into account passenger preferences, conditions on transport links, and projected queues at the airport).

\subsubsection{Operation in disrupted conditions}

We consider a crisis situation as one which results in many flight cancellations at one or more airports. Information about cancellations may become available whilst the passenger is still at home, whilst they are travelling to the airport, or whilst they are waiting at the gate. For passengers on a cancelled flight, all P-CDM successive milestones are interrupted. As soon as information on cancellation is available, the normal milestone process is interrupted and a crisis milestone process begins instead. P-CDM partners (airline, airport, etc.) commit to collaborate (as is already the case in nominal conditions) so as to provide information and solutions 
to each passenger. The direct connection of passengers to the P-CDM system also allows them to contact the system if necessary.

Passengers are given information on the cancellation, on their rights, and on the options that are available to them. These options might include a refund, an alternative flight, or transfer to another mode (either for the full journey, or to an alternative airport for a flight to their destination). This information is tailored to passengers' stated preferences and current location. Once passengers have made a choice between options, they are given practical information on how to proceed. If the passenger chooses to travel (either by an alternative flight or ground transportation) then milestones are calculated for the new journey and the nominal milestone process is restarted.

Figure 7 illustrates how the P-CDM milestone chain works in this case. Some specific P-CDM crisis milestones are added:

MA: Information on flight cancellation is provided by the air transport operator,

MB: Information on the list of options for passengers is provided,

MC: The passenger chooses an option,

MD: Information on practical details relative to the chosen option is provided.

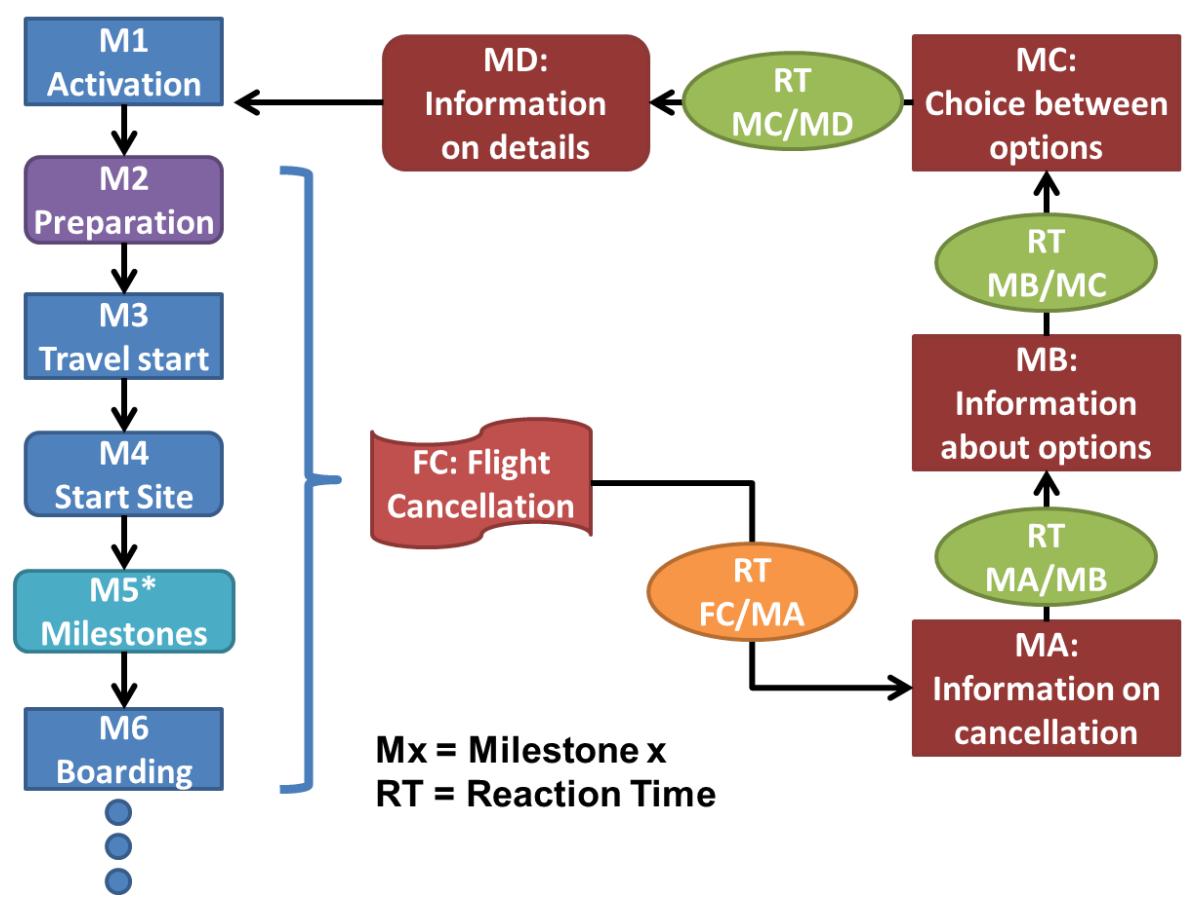




\section{Figure 7: Illustration of the P-CDM milestone chain under disrupted conditions.}

In crisis situations, the time between two successive milestones is difficult or impossible to predict and the notion of variable process and travel time prediction is no longer relevant. Instead, reaction times (RTs) are defined as limits on the maximum time P-CDM partners should spend between two successive milestones:

RT between flight cancellation and Milestone A (RT FC/MA) is the time between the decision to cancel the flight and the provision of the corresponding information to the passenger.

RT between milestones A and B (RT MA/MB) is the time between the information of the flight cancellation and the provision of options to the passenger.

RT between milestones B and C (RT MB/MC) is the time between the provision of options to the passenger and the choice between options made by the passenger.

RT between milestones $\mathrm{C}$ and $\mathrm{D}$ (RT MC/MD) is the time between the passenger choice of options and the provision of practical details relative to this choice.

These RTs allow passengers to anticipate when they should have more information from the P-CDM system.

To identify the characteristics of the different alternative options that can be offered to the passenger during RT $\mathrm{MA} / \mathrm{MB}$, information needs to be exchanged between passengers, airlines, ground transport operators and hotels. Once the passenger chooses an option, they additionally need to be provided with practical assistance to take that option (reimbursement, alternative flight ticket, hotel room voucher, train ticket, etc.) as required, including all the needed additional information to guide the passenger toward the next step (e.g. new airport gate, station platform, etc.). Co-ordination between the different partners, and between the partners and passengers, is handled by the central P-CDM system, using similar collaborative protocols to those used in ACDM. Hence, the airline can easily communicate with the other partners when determining the alternative options to be provided to the passenger. Once the options are identified, the airline sends them, through the system, to the passenger who then has to choose one.

Introducing ground transportation into this process also adds a layer of legal complexity which would need to be addressed in the design of any system. For example, passenger rights in the case of disruption or an accident whilst using ground alternatives would need to be clarified. Whilst passengers who do not want to use P-CDM would still have the same rights as at present, the rights of passengers using P-CDM would need to be clarified 
(for example, does use of P-CDM count towards the legal compensation requirements for delayed passengers?

What rights do passengers who abandon a P-CDM journey in the middle have?).

The options available to passengers will necessarily differ by passenger circumstance, for example:

1. The characteristics of the cancelled flight for the passenger: outbound flight, inbound or connecting flight,

2. The length of the cancelled flight: short-haul flight vs. medium or long-haul flight,

3. The state of alternative transport modes (are they also affected by the disruptive event?),

4. Which airports are affected by the disruption (the departure airport, the arrival airport, and/or any nearby alternative airports with flights to the arrival airport), and

5. Whether the passengers are able and willing to make a ground transport journey (do they have right of entry to all countries en route, and is the ground route compatible with any accessibility requirements they may have?)

For example, a passenger who needs to catch a connecting flight could be given priority on an alternative flight, if it will enable them to meet their connection; if there is no alternative that will allow meeting the connection, then they may be a candidate for a slower mode alternative which will meet the next available connection, or their whole itinerary could be rebooked (for example, by using a train to get to a different hub airport). One significant challenge here is that each disrupted situation is unique, requiring an adaptive response in each case and limiting future learning opportunities.

\section{CONCLUSIONS}

The recovery period from disruptive events at airports can be unpleasant both for passengers and airlines. The work carried out in this paper demonstrates that, for disruptive events where at least some ground transportation is still functional, transferring some stranded passengers to ground transport modes, other air carriers or other nearby airports to help them reach their destination sooner could be a plausible option. Although there are logistical barriers, many are similar to those that apply in other situations where airlines and other potentially competing stakeholders have to work together to produce an optimised outcome. Therefore, an approach 
adapted from the airport CDM framework, which has already begun to address these types of problems, is suggested here. The increasing numbers of travellers carrying smartphones allows passengers to participate in this information exchange framework. However, any CDM approach would need significant adaptation to cope with the increased number and diversity of stakeholders involved, and the different types of disruption.

Our analysis of this scenario in practice suggests that it could be cost-effective for airlines if around 10-50\% of stranded passengers could be routed via alternative methods, depending on the airport and the length and type of disruption experienced. Compared to the current standard of a later flight by the same carrier, airline passenger delay-related costs could be reduced by up to $20 \%$, and mean passenger delay by up to $70 \%$. This occurs mainly via a reduction in very long delays. We do not find any one alternative option dominating over the others; rather, the lowest-cost solution employs a portfolio of different methods to get stranded passengers to their final destination depending on the route and associated journey times and costs. Although the exact portfolio of methods depends on assumptions about the cost and time associated with each method, our sensitivity study finds that the overall cost benefits are relatively insensitive to these assumptions, although they are sensitive to assumptions about airline cost of passenger delay.

These outcomes suggest that there is scope for the current, limited use of these methods to be significantly expanded. This applies particularly at airports which have the advantage of good regional transport links, and at airports where utilisation close to capacity and/or high load factors mean that disruption recovery via traditional methods is more difficult. In contrast, airports in geographically isolated positions may stand to see relatively little benefit. However, in all cases examined in this paper at least a 5.7\% reduction in overall airline passenger delay-related cost was achievable for an airport closure period of 10 hours.

There are a number of limitations to the approach in this paper which could be addressed by future work. Realworld disruptive events normally involve a mix of delayed, cancelled, diverted and unaffected flights rather than a straightforward block of cancellations as used in this paper, and may involve disruption to ground transport as well. Similarly, real passengers may have connecting flights to catch and there may be differences between passenger preferences and/or actions and the airline's cost optimum situation which could be captured with choice modelling. Optimising for cost also requires a good prediction for when the disrupted period will end, which may not be available. Throughout the optimisation, there are many uncertain parameters and a real-world P-CDM framework would need to be able to deal with high levels of uncertainty and data of variable quality. The optimization formulation in this paper is generalisable to the case where multiple airports are disrupted, 
ground transport is also subject to delays, and the impact of disruption on scheduling is more complex than a simple period of airport closure followed by full operation. Therefore significant scope also exists here for future work looking at the best way to respond to disruption by type. For example, the best passenger reaccommodation strategy is likely to be very different for a snow and ice event affecting all London airports, compared to the closure of a single airport, compared to a technical failure which limits throughput but does not lead to full airport closure. In these cases, the decision over which flights to cancel may also form a critical part of an ideal optimisation strategy.

\section{ACKNOWLEDGEMENTS}

MetaCDM, which this research is based on, was an EC FP7-funded project (grant agreement number 314453). The authors are grateful for this support and for the help and assistance of the many people involved in the MetaCDM workshop, interview and data-gathering stages, particularly our project partners Thomas Günther, Gunnar Spies and Roger Gardner.

\section{REFERENCES}

[1] European Commission (EC), 2011. White paper: Roadmap to a single European transport area towards a competitive and resource efficient transport system. http://ec.europa.eu/transport/themes/strategies/2011_white_paper_en.htm [accessed June 2016]

[2] Marks, J., Jenkins, D. (2010). Modeling passenger reaccommodation time for flight cancellations in airline networks, PDM, http://www.tarmaclimits.com/Tarmac/Tarmac Limits_files/Passenger\%20Displacement\%20Paper.pdf [accessed October 2015]

[3] Bratu, S. and Barnhart, C., 2006. Flight operations recovery: New approaches considering passenger recovery. Journal of Scheduling, 9(3), 279-298.

[4] Marzuoli, A., Laplace, I., Feron, E., Dray, L. Gardner, R., Günther, T. and Spies, G., 2013. Analysis of Recent Disruptions of the Air Transport System. MetaCDM WP1 Report, http://www.metacdm.org/Deliverables/META-CDM_D1.2_1.0.pdf [Accessed July 2015]

[5] Eurocontrol, 2010. Ash Cloud of April and May 2010: Impact on Air Traffic. 
http://www.eurocontrol.int/sites/default/files/content/documents/official-documents/facts-and-

figures/statfor/ash-impact-air-traffic-2010.pdf [Accessed June 2016].

[6] Laplace, I., Marzuoli, A., Feron, E., Dray, L., Gardner, R., Günther, T. and Spies, G., 2014. Contributions of Information Sharing, Collaborative Decision Making and Multimodality in improving passenger experience during disruptive events. MetaCDM WP2 Report, http://www.meta-

cdm.org/Deliverables/META_CDM_D2.2 1.0.pdf [Accessed February 2015]

[7] Mukherjee, A., Ball, M. and Subramanian, B., 2006. Models for estimating monthly delays and cancellations in the NAS, in NEXTOR NAS Performance Metrics Conference, Asilomar, Calif, 2006.

[8] Pyrgiotis, N., Malone, K. and Odoni, A., 2011. Modelling delay propagation within an airport network. Transportation Research Part C, 27, 60-75.

Airport Co-operative Research Program (ACRP), 2015. ACRP Report 118: Integrating aviation and passenger rail planning.

[9] Nayak, N., 2012. Estimation of the impact of single airport and multi-airport system delay on the national airspace system using multivariate simultaneous models. Ph.D. dissertation,

University of South Florida.

http://onlinepubs.trb.org/onlinepubs/acrp/acrp_rpt_118.pdf [Accessed December 2016]

[10] Baumgarten, P., Malina, R., \& Lange, A. (2014). The impact of hubbing concentration on flight delays within airline networks: An empirical analysis of the US domestic market. Transportation Research Part E: Logistics and Transportation Review, 66, 103-114.

[11] AhmadBeygi, S., Cohn, A., Guan, Y., and Belobaba, P., 2008. Analysis of the potential for delay propagation in passenger airline networks. Journal of Air Transport Management, 14(5), 221-236.

[12] Jarrah, A. I., Yu, G., Krishnamurthy, N., \& Rakshit, A. (1993). A decision support framework for airline flight cancellations and delays. Transportation Science, 27(3), 266-280.

[13] Bisaillon, S., Cordeau, J.F., Laporte, G. and Pasin, F. (2011). A large neighbourhood search heuristic for the aircraft and passenger recovery problem", Operations Research, 9(2), 139-157.

[14] Jafari, N., and Zegordi, S. H., 2011. Simultaneous recovery model for aircraft and passengers. Journal of the Franklin Institute, 348(7), 1638-1655.

[15] Petersen, J. D., Solveling, G., Clarke, J.-P., Johnson, E. L., and Shebalov, S., 2012. An optimization approach to airline integrated recovery. Transportation Science, 46(4), 482-500. 
[16] Kim, S. H., Feron, E., Clarke, J. P., Marzuoli, A., \& Delahaye, D. (2013). Airport Gate scheduling for passengers, aircraft, and operation. arXiv preprint arXiv:1301.3535.

[17] Chung, S. H., Tse, Y. K., \& Choi, T. M. (2015). Managing disruption risk in express logistics via proactive planning. Industrial Management \& Data Systems, 115(8), 1481-1509.

[18] Ball, M., 2006. Analysis of passenger delays: Developing a passenger delay metric, in NEXTOR NAS Performance Metrics Conference, Asilomar, CA, 2006.

[19] Wang, D., 2007. Methods for analysis of passenger trip performance in a complex networked transportation system. Ph.D. dissertation, George Mason University.

[20] Cook, A., Tanner, G., Cristóbal, S. and Zanin, M., 2014. Final Technical Report, POEM project.

[21] World Economic Forum (WEF), 2013. Connected world: transforming travel, transportation and supply chains. http://www3.weforum.org/docs/WEF_MO_ConnectedWorld_Report_2013.pdf [accessed January 2015]. [22] WEF, 2014. Smart travel: Unlocking economic growth and development through travel facilitation. http://www3.weforum.org/docs/GAC/2014/WEF_GAC_TravelTourism_SmartTravel_WhitePaper_2014 [accessed January 2015].

[23] Zhang, Y. and Hansen, M. 2008. Real-time intermodal substitution strategy for airline recovery from schedule perturbation and for mitigation of airport congestion. Transportation Research Record, 2052, 90-99. [24] Dray, L., Marzuoli, A., Evans, A., Laplace, I., \& Feron, E. (2015, June). Air Transportation and Multimodal, Collaborative Decision Making during Adverse Events. In ATM seminar 2015, 11th USA/EUROPE Air Traffic Management $R \& D$ Seminar.

[25] Marzuoli, A. C. (2015). Multimodal Collaborative Passenger-centric Decision Making to Mitigate the Impact of Airside Perturbations. Thesis, Georgia Institute of Technology, April 2015.

https://smartech.gatech.edu/handle/1853/53578 [Accessed December 2016]

[26] Marzuoli, A., Laplace, I., Feron, E., Dray, L., Gardner, R., Spies, G. and Günther, T., 2014. MetaCDM Concept of Operations. http://www.meta-cdm.org/Deliverables/MetaCDM_WP3_D3.2_2.0.pdf [Accessed December 2016]

[27] Airports Council International (ACI), 2014. Passenger Traffic 2012. http://www.aci.aero/DataCentre/Annual-Traffic-Data/Passengers/2012-final

[28] Official Airline Guide (OAG), 2012. Global Flight Schedules for 2012. http://www.oagdata.com.

[29] Eurostat, 2014. Air Passenger and Vehicle Stock Statistics Databases.

http://epp.eurostat.ec.europa.eu/portal/page/portal/transport/data/database. 
[30] Dray, L., Evans, A., Reynolds, T. and Schäfer, A., 2010. Mitigating Aviation Carbon Dioxide Emissions: An Analysis for Europe. Transportation Research Record, 2177, 17-26.

[31] ETISplus, 2014. European Transport Policy Information System database.

http://www.etisplus.eu/default.aspx

[32] Deutsche Bahn, 2014. Bahn.de Online Journey Planner. http://www.bahn.de

[33] Andersson, E., Berg, M., Nelldal, B.-L., \& Fröidh, O., 2011. TOSCA WP3 Final Report. http://www.toscaproject.org/FinalReports/TOSCA_WP3_RailPassenger.pdf

[34] HM Revenue and Customs (HMRC), 2014. Approved mileage and fuel allowances. http://www.hmrc.gov.uk/rates/travel.htm.

[35] MapQuest, 2014. MapQuest Online Journey Planner. http://www.mapquest.com.

[36] TravelMath, 2014. TravelMath Online Journey Planner. http://www.travelmath.com.

[37] Maertens, S., Grimme, W., 2015. How to assess the percentage of transfer passengers at airports? German Aerospace Center Discussion Paper, June 2015,

http://www.dlr.de/fw/Portaldata/42/Resources/dokumente/paper/Maertens_Grimme_Transfer_Rate_estimation.p df [Accessed December 2016]

[38] Xiong, J., \& Hansen, M. (2013). Modelling airline flight cancellation decisions. Transportation Research Part E: Logistics and Transportation Review, 56, 64-80.

[39] Bravo, H. C., 2014. Package 'Rcplex'. http://cran.r-project.org/web/packages/Rcplex/Rcplex.pdf. [Accessed November 2015]

[40] European Telecommunications Standards Institute (ETSI), 2010. “Airport collaborative Decision Making (A-CDM); Single European Sky Interoperability Regulation EC 552/2004”, ETSI EN 303 212, Version 1.1.1, June 2010

[41] Société Internationale de Télécommunications Aéronautiques (SITA), 2013. Passengers say 'yes’ to technology but mobile usage still low. News release, October 2013. http://www.sita.aero/pressroom/news$\underline{\text { releases/passengers-say-yes-to-technology-but-mobile-usage-still-low [Accessed December 2016] }}$

[42] Eurocontrol, 2016. A-CDM Impact Assessment. https://www.eurocontrol.int/sites/default/files/publication/files/a-cdm-impact-assessment-2016.pdf [Accessed December 2016] 


\section{Appendix A - Mathematical Formulation of Optimization Problem}

In this appendix, we present the mathematical formulation to solve the optimization problem described in Section 2.2. This is preceded by a detailed description of the variables used in the formulation in Section A.1, and followed by a description of the formulation in Section A.3.

\section{A.1 Definition of Variables}

$A$

- Number of destination airports served by the disrupted airport.

$B_{j, c, z} \quad-$ Number of coaches of size $z$ to airport $j$, on which passengers on cancelled flight $c$ can be accommodated.

$C($ ) $\quad-$ Hard and soft airline costs, per passenger, as a function of passenger delay (the cost of providing food, accommodation and other services for stranded passengers, and the costs incurred due to disgruntled passengers switching to other airlines in future, respectively).

$\mathrm{CBH}_{j, z} \quad-$ The cost of hiring a coach (bus) of size $z$ to transport passengers to airport $j$.

$C R F_{k, j, f} \quad-$ The cost of re-accommodating passengers from airport $k$ to airport $j$ on flight $f$ (defined explicitly in equation $\mathrm{A} 2) . C R F_{0, j, f}$ refers to the cost of re-accommodating passengers from the disrupted airport to airport $j$.

$C R T_{j, t} \quad-$ The cost of a ticket (to be paid by the airline) to airport $j$ on train $t$.

$c_{t o t j} \quad-$ Number of cancelled flights to airport $j$.

$D A B_{j, c, k, z b, f} \quad$ - Arrival delay of the alternate routing by coach to airport $j$, through airport $k$, relative to the scheduled arrival time of cancelled flight $c$. Coach (bus) $b$ of size $z$ is operated from the disrupted airport to airport $k$, and flight $f$ is operated from airport $k$ to airport $j$.

$D A T_{j, c, k, t, f} \quad$ - Arrival delay of the alternate routing by train to airport $j$, through airport $k$, relative to the scheduled arrival time of cancelled flight $c$. Train $t$ is operated from the disrupted airport to airport $k$, and flight $f$ is operated from airport $k$ to airport $j$.

$D B_{j, c, z, b} \quad-$ Arrival delay of coach $b$, of size $z$, to airport $j$, relative to the scheduled arrival time of cancelled flight $c$. 
$D F_{j, c, f} \quad-$ Arrival delay of flight $f$ to airport $j$, relative to the scheduled arrival time of cancelled flight $c$.

$D T_{j, c, f} \quad-$ Arrival delay of direct train $t$ to airport $j$, relative to the scheduled arrival time of cancelled flight $c$.

$F_{j, c} \quad-$ Number of alternate flights to airport $j$, on which passengers on cancelled flight $c$ can be accommodated.

Fare $_{k, j, f} \quad-$ Average airfare for flight $f$ from airport $k$ to airport $j$. Fare $_{0, j, f}$ refers to the airfare for flight $f$ from the disrupted airport to airport $j$.

$K_{j, c} \quad-$ The number of alternate airports that can be reached by train or coach (bus) from the disrupted airport, that have flights to airport $j$, on which passengers could be re-accommodated from cancellation $c$.

$N B_{j, c, z}$

- The number of coaches (buses) of size $z$ hired to transport passengers from cancelled flight $c$ to airport $j$.

- The total regional coach (bus) stock (all sizes).

$P_{C A} \quad-$ The fraction of coaches (buses) available for hire.

$P_{R O} \quad-$ The fraction of cancelled passengers who choose to be reimbursed and either not fly or be returned to their point of origin.

$\operatorname{Pax}_{j, c} \quad-$ Number of passengers flying to airport $j$ on cancelled flight $c$.

$\operatorname{PaxAB}_{j, c, k, z, b, f} \quad$ - Number of passengers travelling to airport $j$ on cancelled flight $c$ that are re-accommodated through airport $k$ on coach $b$ of size $z$ from the disrupted airport to airport $k$, and flight $f$ from airport $k$ to airport $j$.

$\operatorname{PaxAT}_{j, c, k, t, f} \quad$ - Number of passengers travelling to airport $j$ on cancelled flight $c$ that are re-accommodated through airport $k$ on train $t$ from the disrupted airport to airport $k$, and flight $f$ from airport $k$ to airport $j$.

$\operatorname{PaxB}_{j, c, z, b} \quad-$ Number of passengers travelling to airport $j$ on cancelled flight $c$ that are re-accommodated on coach $b$, of size $z$.

$\operatorname{PaxF}_{j, c, f} \quad-$ Number of passengers flying to airport $j$ on cancelled flight $c$ that are re-accommodated on alternate flight $f$.

$\operatorname{PaxT}_{j, c, t} \quad-$ Number of passengers travelling to airport $j$ on cancelled flight $c$ that are re-accommodated on direct train $t$.

$S B_{z} \quad-$ The number of seats on a coach (bus) of size $z$. 

$S F_{j, f}$
- The number of empty seats available on flight $f$ to airport $j$.
$S T_{j, t} \quad-$ The number of empty seats available on train $t$ to airport $j$.
$T_{j, c} \quad-$ Number of direct trains to airport $j$, on which passengers on cancelled flight $c$ can be accommodated.
Z
- The number of coach sizes.

\section{A.2 Optimization Formulation}

The optimization problem is formulated as follows:

\section{Objective Function:}

$$
\begin{aligned}
& \min \left(\sum _ { j = 1 } ^ { A } \sum _ { c = 1 } ^ { c _ { \text { tot } j } } \left[\operatorname{Fare~}_{0, j, c} \cdot P_{R o} \cdot \operatorname{Pax}_{j, c}+\sum_{f=1}^{F_{j, c}}\left(\operatorname{PaxF}_{j, c, f} \cdot\left(C\left(\operatorname{DF}_{j, c, f}\right)+C R F_{0, j, f}\right)\right)\right.\right. \\
& +\sum_{t=1}^{T_{j, c}}\left(\operatorname{PaxT}_{j, c, t} \cdot\left(C\left(D T_{j, c, t}\right)+C R T_{j, t}\right)\right) \\
& +\sum_{z=1}^{Z}\left(\sum_{b=1}^{B_{j, c, z}}\left(\operatorname{Pax}_{j, c, z, b} \cdot C\left(D B_{j, c, z, b}\right)\right)+N B_{j, c, z} \cdot C B H_{j, z}\right) \\
& +\sum_{k=1}^{K_{j, c}} \sum_{t=1}^{T_{k, c}} \sum_{f=1}^{F_{k, j, c}}\left(\operatorname{PaxAT}_{j, c, k, t, f} \cdot\left(C\left(D A T_{j, c, k, t, f}\right)+C R T_{k, t}+C R F_{k, j, f}\right)\right) \\
& +\sum_{k=1}^{K_{j, c}} \sum_{z=1}^{Z}\left(\sum_{b=1}^{B_{k, c, z}} \sum_{f=1}^{F_{k, j, c}}\left(\operatorname{Pax} A B_{j, c, k, Z, b, f} \cdot\left(C\left(D A B_{j, c, k, z, b, f}\right)+C R F_{k, j, f}\right)\right)\right. \\
& \left.\left.\left.+N B_{k, c, z} \cdot C B H_{k, z}\right)\right]\right)
\end{aligned}
$$

\section{Decision variables:}

$\operatorname{PaxF}_{j, c, f} ; \operatorname{PaxT}_{j, c, t} ; \operatorname{Pax}_{j, c, z, b} ; \operatorname{PaxAT}_{j, c, k, t, f} ; \operatorname{PaxAB}_{j, c, k, z, t, f} ; N B_{j, c, z} ; N B_{k, c, z}$ 


\section{Subject to:}

$$
C R F_{i, j, f}=\left\{\begin{array}{cc}
0 & \text { if } f \text { is a later flight from } i \text { to } j \text { by an aligned airline } \\
\text { Fare }_{i, j, f} & \text { if } f \text { is a later flight from } i \text { to } j \text { by a non aligned airline }
\end{array}\right.
$$

for each $i \in\{1, \ldots, A\} ; j \in\{1, \ldots, A\} ; c \in\left\{1, \ldots, c_{t o t}\right\} ;$ and $f \in\left\{1, \ldots, f_{\text {tot }}\right\}$

$$
\begin{aligned}
\operatorname{Pax}_{j, c}\left(1-P_{R O} / 2\right) & \\
= & \sum_{f=1}^{F_{j, c}} \operatorname{PaxF}_{j, c, f}+\sum_{t=1}^{T_{j, c}} \operatorname{Pax}_{j, c, t}+\sum_{z=1}^{Z} \sum_{b=1}^{B_{j, c, z}} \operatorname{Pax}_{j, c, z, b}+\sum_{k=1}^{K_{j, c}} \sum_{t=1}^{T_{k, c}} \sum_{f=1}^{F_{k, j, c}} \operatorname{PaxAT}_{j, c, k, t, f} \\
& +\sum_{k=1}^{K_{j, c}} \sum_{z=1}^{Z} \sum_{b=1}^{B_{k, c, z} F_{k, j, c}} \sum_{f=1}^{P} \operatorname{PaxAB_{j,c,k,z,t,f}}
\end{aligned}
$$

for each destination $j \in\{1, \ldots, A\}$; and cancelled flight $c \in\left\{1, \ldots, c_{\text {tot }}\right\}$

$$
\sum_{c=1}^{c_{t o t} i, j} \operatorname{Pax} F_{j, c, f} \leq S F_{j, f}
$$

for each destination $j \in\{1, \ldots, A\}$; and alternate flight $f \in\left\{1, \ldots, F_{j, c}\right\}$

$$
\sum_{c=1}^{c_{\text {tot } j}} \operatorname{PaxT}_{j, c, t}+\sum_{k=1}^{A} \sum_{c=1}^{c_{\text {tot }}} \sum_{f=1}^{F_{j, k, c}} \operatorname{PaxAT}_{k, c, j, t, f} \leq S T_{j, t}
$$

for each destination $j \in\{1, \ldots, A\}$; and train $t \in\left\{1, \ldots, T_{j, c}\right\}$

$$
\sum_{c=1}^{c_{\text {tot } j}} \sum_{b=1}^{B_{j, c, z}} \operatorname{PaxB}_{j, c, z, b}+\sum_{k=1}^{A_{D}} \sum_{c=1}^{c_{\text {tot }}} \sum_{b=1}^{B_{j, c, z}} \sum_{f=1}^{F_{j, k, c}} \operatorname{PaxA} B_{k, c, j, z, b, f} \leq \sum_{c=1}^{c_{\text {tot } j}} S B_{z} \cdot N B_{j, c, z}
$$

for each destination $j \in\{1, \ldots, A\}$; and coach size category $z \in\{1, \ldots, Z\}$ 


$$
\sum_{j=1}^{A} \sum_{c=1}^{c_{\text {tot } j}} N B_{j, c, z}+\sum_{k=1}^{K_{j}} \sum_{c=1}^{c_{\text {tot } j}} N B_{k, c, z} \leq N B_{z}
$$

for each coach size category $z \in\{1, \ldots, Z\}$

$$
N B_{z}=P_{C A} \cdot \frac{N B R}{Z}
$$

for each coach size category $z \in\{1, \ldots, Z\}$

\section{A.3 Description of Optimization Formulation}

The objective function, shown in Equation A-1, is a minimization of the summation of airline costs across all cancelled flights scheduled from the disrupted airport to all destination airports. Airline costs consist of six different terms. These are the cost of reimbursing passengers who choose not to fly or be returned to their point of origin (depending on whether they are at their point of origin or not), and the cost associated with five possible options for re-accommodating the disrupted passengers. The re-accommodation options considered in this paper are: (1) later flight to the destination (by the same airline, an alliance partner or a non-aligned airline);

(2) train to the destination city; (3) coach to the destination city; (4) train to an alternative airport followed by a flight to the final destination; and (5) coach to an alternative airport followed by a flight to the final destination.

The cost of reimbursing passengers who choose not to fly or be returned to their point of origin is as sumed to equal the average fare paid for the cancelled flight, as shown in the first term of Equation A-1. The cost of reaccommodating passengers on each of the options described above includes two terms: (1) the cost associated with the passenger delay relative to their scheduled arrival time on the delayed flight, and (2) the cost of the alternate transport option. In the case of alternative flight options, this latter cost can be zero, if the flight is operated by an aligned airline, or equal to the average fare paid for the alternate flight, if the flight is not operated by an aligned airline, as described in Equation A-2.

Decision variables in the optimisation include the number of passengers that are re-accommodated on each reaccommodation option, between each origin destination pair, for each cancellation, as well as the number of 
coaches hired for the two re-accommodation options that use coaches (a coach from the origin to the destination city, and a coach to an alternative airport, followed by a flight to the final destination).

Seven constraints are included in the optimization. The first equates the cost of the alternative flights to either zero, if the flight is operated by an aligned airline, or to the average fare paid for the alternate flight, if the flight is not operated by an aligned airline (Equation A-2).

The second constraint (equation A-3) ensures that the total number of passengers on the cancelled flights who choose to be re-accommodated equals the total number of passengers re-accommodated on each option, i.e., a later flight; a train to the destination city; a coach to the destination city; a train to an alternative airport followed by a flight to the final destination; and a coach to an alternative airport followed by a flight to the final destination.

The third, fourth and fifth constraints (equations A-4, A-5 and A-6) ensure that the number of passengers reaccommodated on each option is less than or equal to the number of seats available on that option. Equation A-4 applies this constraint for flights, with the term on the left-hand-side referring to the total number of passengers being re-accommodated on a later non-stop flight to the destination airport. If only one airport $i$ is disrupted, then these two latter terms are zero. The right-hand-side of the equation is the number of seats available on flight $f$ from airport $i$ to airport $j$.

Equation A-5 applies the seat constraint for trains. Similar to equation A-4, the first term on the left-hand-side refers to the total number of passengers being re-accommodated on a train to the destination city. The second term on the left-hand-side accounts for disrupted passengers travelling to other destinations $k$, but who take a train to airport $j$ before boarding a flight to their final destination $k$. Similarly to equation A-4, the right-handside of the equation is the number of seats available on train $t$ to airport $j$.

Equation A-6 applies the seat constraint for coaches. Similarly to equations A-4 and A-5, the first term on the left-hand-side refers to the total number of passengers being re-accommodated on coaches to the destination city. The second term on the left-hand-side accounts for disrupted passengers travelling to other destinations $k$, but who take a coach to airport $j$ before boarding a flight to their final destination $k$. The right-hand-side of the equation is the number of seats available on all the coaches to airport $j$. This latter term takes a different form to the right-hand-side in equations A-4 and A-5 because the number of coaches the airline hires is also a decision variable. 
The sixth constraint (equation A-7) ensures that the number of coaches of each size hired to take passengers to all destinations is less than or equal to the total number of coaches of that size available for hire. The first term on the left-hand-side accounts for passengers travelling to their final destination by coach, while the second term accounts for passengers travelling to an alternate airport by coach, before taking a flight to their final destination.

The final constraint (equation A-8) calculates the number of coaches of each size available at the origin airport to be equal to the product of the total number of coaches available in the region, divided by the number of aircraft size categories, and an assumed availability fraction $\left(P_{C A}\right)$. 